\title{
Results From the Galileo Giove-A Radiation Monitors and Comparison With Existing Radiation Belt Models
}

\author{
Ben Taylor, Member, IEEE, Craig I. Underwood, Hugh D. R. Evans, Keith Ryden, David Rodgers, \\ Eamonn J. Daly, Giuseppe Mandorlo, Marco Falcone, P. A. Morris, and Rafael Garcia Prieto
}

\begin{abstract}
The radiation monitors on board the Galileo Giove-A satellite, CEDEX and Merlin, and their data are presented. The instruments include energetic proton and ion detectors, an internal charging monitor, RADFETs and experimental dose-rate photodiodes. A comparison of the data with existing monitors and models is presented.
\end{abstract}

Index Terms-Electron, Galileo, GIOVE-A, GSTB-V2, integral, ionising dose, IREM, LET, proton, radiation environment, radiation monitor.

\section{INTRODUCTION}

$\mathbf{I}$ $\mathrm{N}$ preparation for the Galileo system, the European Space Agency commissioned two test satellites, the first of which is the Giove-A, built by Surrey Satellite Technology. These satellites will test the technology required for the Galileo System and characterise the space environment that the final constellation will encounter. Giove-A was launched on the 28th December 2005 into a $23260 \mathrm{~km}$ circular orbit with an inclination of $56^{\circ}$. The second, Giove-B, built by Galileo Industries, is planned for launch in 2007, and will carry a SREM instrument [1]. To characterise the environment, Giove-A's payload includes two radiation monitors: CEDEX (Univ. Surrey) and MERLIN (QinetiQ).

The Cosmic-Ray Energy Deposition Experiment (CEDEX) monitors high-energy $(45-50 \mathrm{MeV})$ proton fluxes and provides a detailed LET spectrum for the cosmic-ray ion environment with a pair of $300 \mu \mathrm{m}$ silicon PIN diode detectors arranged to form a telescope. In addition, CEDEX carries four experimental dose-rate sensors, shielded by domes of thickness $2 \mathrm{~mm} \mathrm{Al}$, $4 \mathrm{~mm} \mathrm{Al}, 2 \mathrm{~mm} \mathrm{Cu}$, and $4 \mathrm{~mm} \mathrm{Cu}$.

Manuscript received October 6, 2006; revised January 10, 2007.

B. Taylor and C. I. Underwood are with the Surrey Space Centre, University of Surrey, Guildford, Surrey GU2 7XH, U.K. (e-mail: B.Taylor@surrey.ac.uk; C.Underwood@surrey.ac.uk).

H. D. R. Evans is with the European Space Agency, ESTEC/TEC-EES, Postbus 299, 2200 AG Noordwijk, The Netherlands and also with the Rhea System SA, Louvain-la-Neuve B-1348, Belgium (e-mail: Hugh.Evans@ esa.int).

K. Ryden, D. Rodgers, and P. A. Morris are with the QinetiQ Space Division, Cody Technical Park, Farnborough GU14 OLX, U.K. (e-mail: KARYDEN@ qinetiq.com; djrodgers@space.qinetiq.com; pamorris@ qinetiq.com).

E. J. Daly, G. Mandorlo, M. Falcone, and R. G. Prieto are with the European Space Agency, ESTEC/TEC-EES, Postbus 299, 2200 AG Noordwijk, The Netherlands (e-mail: Eamonn.Daly@esa.int; Giuseppe.Mandorlo@esa.int; Marco.Falcone@esa.int; Rafael.Garcia.Prieto@esa.int).

Color versions of one or more of the figures in this paper are available online at http://ieeexplore.ieee.org.

Digital Object Identifier 10.1109/TNS.2007.892115

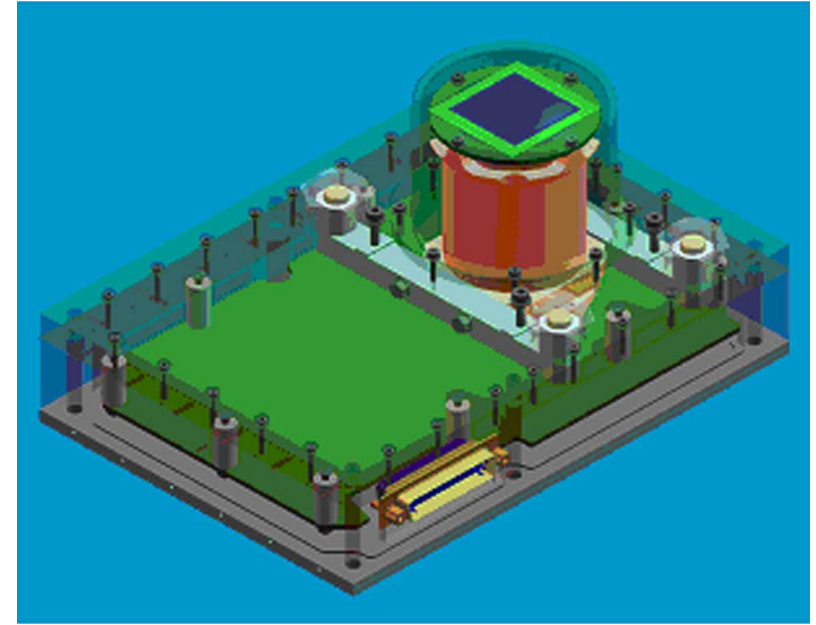

Fig. 1. The University of Surrey's CEDEX instrument.

Merlin includes a PIN diode detector to measure $>40 \mathrm{MeV}$ proton fluxes, a LET telescope for measuring cosmic rays, two RADFETs and the SURF instrument for measuring energetic electrons and monitoring electrostatic charging.

\section{CEDEX InSTRUMENT}

The Giove-A CEDEX (as seen in Fig. 1) is a development of the TiungSAT-1 CEDEX payload, which was launched in 2000 into low-Earth orbit. This payload in turn has flight heritage through Surrey's series of Cosmic-Ray Experiment (CRE) payloads flown on KITSAT-1 (1992), PoSAT-1 (1993) and AMSAT-OSCAR-40 (2000) [2], [3]. CEDEX's mass is $2.05 \mathrm{~kg}$ (including radiation shielding) and its power consumption is $4.2 \mathrm{~W}$ at $38 \mathrm{~V}$.

\section{A. Proton/Heavy Ion Telescope}

The CRE and CEDEX payloads each detect single-event effect (SEE)-inducing particles (protons and heavy ions) by means of PIN diode detectors. Particles passing through the detector lose energy by creating electron-hole pairs. The resulting charge is proportional to the total energy deposited by the particle, which is itself related to the particle's Linear Energy Transfer (LET). This charge is measured, and a running total is kept of the number of particle events in each charge or LET "bin". 


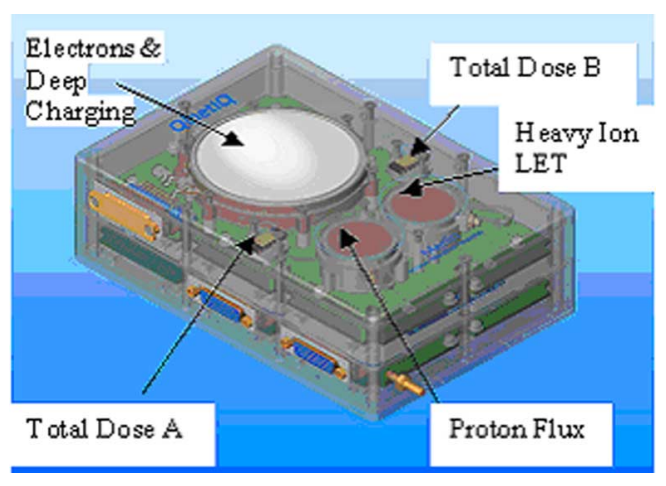

Fig. 2. The QinetiQ Merlin instrument.

The sensing elements comprise two precision $3 \mathrm{~cm} \times 3 \mathrm{~cm}$ PIN diodes at $74 \mathrm{~mm}$ spacing, mounted co-axially to give a $44^{\circ}-60^{\circ}$ field-of-view particle telescope. The upper diode acts as a coincidence detector, whilst the lower diode is connected to a 512-channel charge pulse-height analyser, capable of processing up to 200000 events per second. The vertical incidence LET range is $32 \mathrm{MeV} \mathrm{cm} \mathrm{g}^{-1}$ to $>10000 \mathrm{MeV}$ $\mathrm{cm}^{2} \mathrm{~g}^{-1}$. Counts in the first 3 channels (up to $\sim 72 \mathrm{MeV}$ $\mathrm{cm}^{2} \mathrm{~g}^{-1}$ —equivalent to $6 \mathrm{MeV}$ of energy deposited in the detector) are taken to be primarily due to protons, whereas events in the channels representing LETs of $95 \mathrm{MeV} \mathrm{cm} \mathrm{g}^{-1}$ and above are taken to be due to heavier ions only. Possible contamination in the proton channels due to heavy ions is under investigation. Coincident and non-coincident events (i.e., those that do not trigger the front detector) are recorded separately. CEDEX particle telescope data are integrated over 75 second periods and are sent to the spacecraft's central on-board computer every five minutes.

\section{B. Experimental Dose-Rate Photo-Diodes}

The dose-rate diodes comprise small PIN diodes configured to give a direct photo current output, the dark current of which provides the dose-rate measurement. The diodes are placed behind domes of aluminium ( $2 \mathrm{~mm}$ and $4 \mathrm{~mm}$ ) and copper ( $2 \mathrm{~mm}$ and $4 \mathrm{~mm}$ ) to give a representative series of measurements of the dose-rate inside the spacecraft at different shielding depths. The diodes are commercial UV-enhanced photodiodes, with the quartz window left in place.

The dark current from these diodes is partly due to the temperature of the diodes, leakage currents in the amplifiers and the presence of ionising particles inducing a current due to the ionising dose-rate. The thermal response of the diodes was taken during Thermal Vacuum Testing, together with the CEDEX temperature sensor. The Engineering Model (EM) of CEDEX and complementary photo-diodes were tested with the $\mathrm{Sr}^{90}$ source in the REEF facility at QinetiQ to determine their response to radiation.

\section{Merlin InSTRUMent}

Merlin (as seen in Fig. 2) is a space weather hazard monitor, which provides a comprehensive space environment monitoring capability on Giove-A. Merlin measures key parameters of the

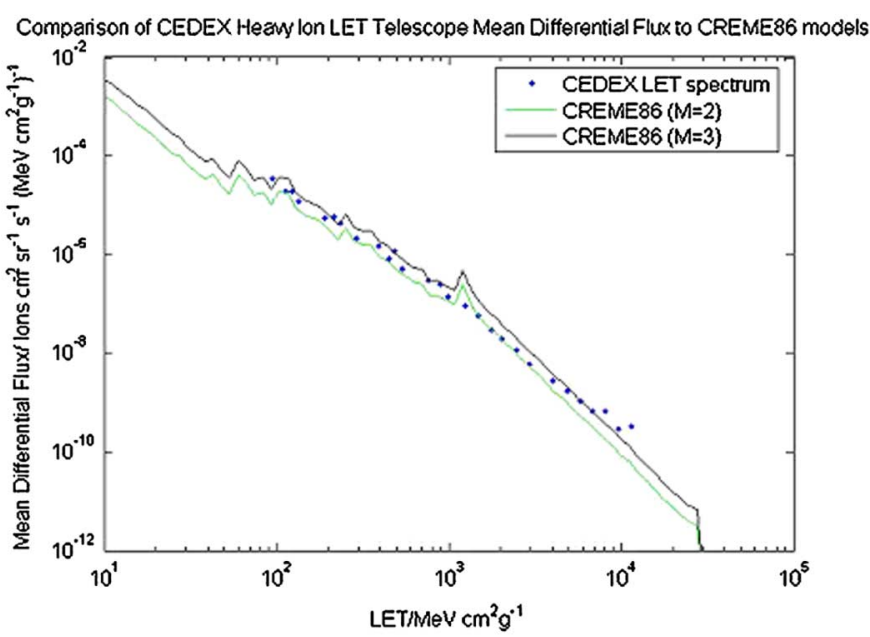

Fig. 3. The mean differential flux spectrum as measured by CEDEX between early January 2006 and late August 2006 overlaid with CREME86 model predictions.

space environment which have practical importance for spacecraft in medium Earth orbit (MEO), namely: internal charging currents, energetic proton fluxes, ion LET spectra and total ionising dose rates in silicon.

Merlin is based on two unique but highly successful instruments, SURF and CREDO, which have flown before on orbits passing through the MEO regime [4]. These two instruments have been repackaged into a $1 \mathrm{~kg}$ unit with combined data handling and power conditioning. For the Giove-A mission an extra interface stage was needed for the CAN bus communications and also additional box level radiation shielding, so giving a 2 $\mathrm{kg}$ overall mass.

\section{A. Particle Telescopes}

Merlin provides two particle telescopes, one for heavy ion LET measurements and one for proton counting. The telescopes comprise two large-area planar silicon diodes separated by $25 \mathrm{~mm}$. Pulse height analysis is applied to determine LET values, and simple thresholds applied for particle counting purposes. The telescope arrangement provides three main benefits: directionality, which enables the particle flux per steradian to be determined; clearer measurements of LET by constraining the angles at which the measured particles pass through the silicon; and better discrimination of the particles of interest against contamination effects from energetic electrons.

\section{B. SURF Monitor}

The SURF monitor is configured to measure internal charging currents in three $70 \mathrm{~mm}$ diameter detector plates stacked one on top of the other. The top two plates are $0.5 \mathrm{~mm}$ thick, and the bottom plate is $1 \mathrm{~mm}$ thick. The combined shielding of the sensor cover and thermal blankets contribute to additional shielding of $0.5 \mathrm{~mm}$ equivalent Aluminium on top of the detector plates. For each plate there is a high-sensitivity and lowsensitivity channel, providing a wide dynamic range. The lowsensitivity channel provides a (dynamic) output of $10 \mathrm{mV} / \mathrm{pA}$, and the high-sensitivity (dynamic) output is $500 \mathrm{mV} / \mathrm{pA}$. This 


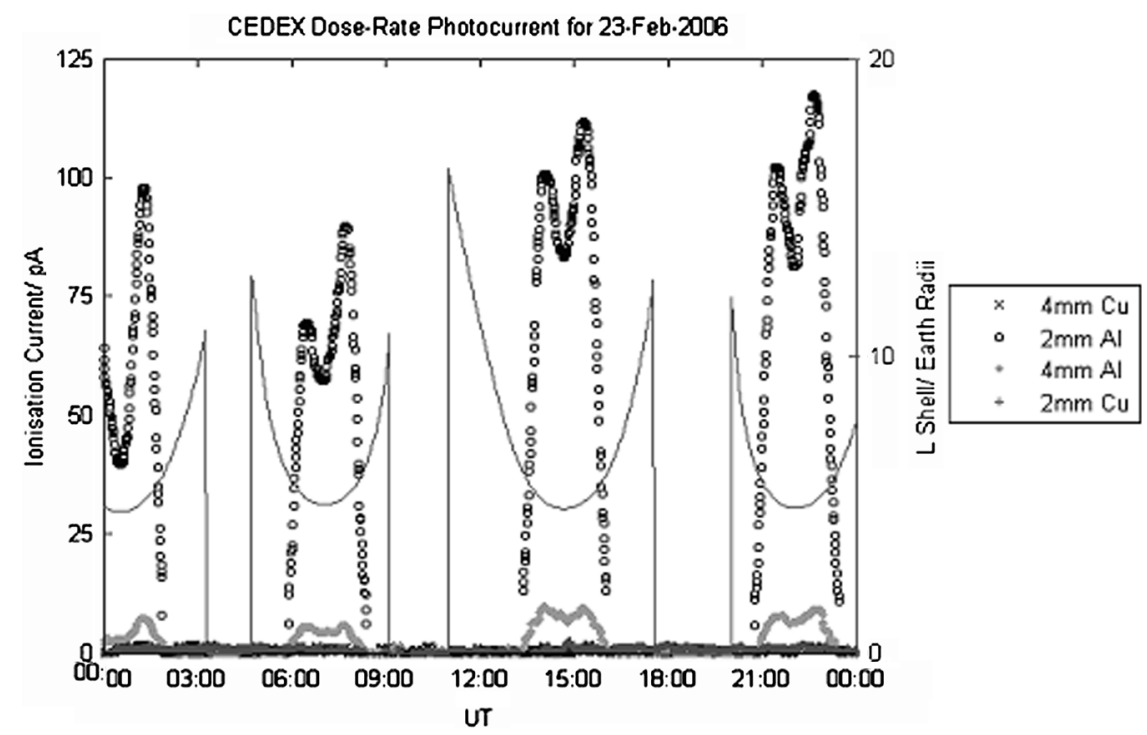

Fig. 4. The Ionisation Currents of the photo diodes from CEDEX plotted against Universal Time for 23 February 2006, overlaid with the McIlwain L shell Geomagnetic coordinate. The signal to noise ratio of the $2 \mathrm{~mm} \mathrm{Al} \mathrm{diode} \mathrm{is} \mathrm{quite} \mathrm{good,} \mathrm{and} \mathrm{the} \mathrm{effects} \mathrm{of} \mathrm{the} \mathrm{radiation} \mathrm{belt} \mathrm{quite} \mathrm{clear.} \mathrm{The} \mathrm{lower} \mathrm{dose} \mathrm{deposition}$ in the $4 \mathrm{~mm} \mathrm{Al}$ diode, though, has a lower signal to noise ratio, but the effects of the radiation belt are still clearly apparent.

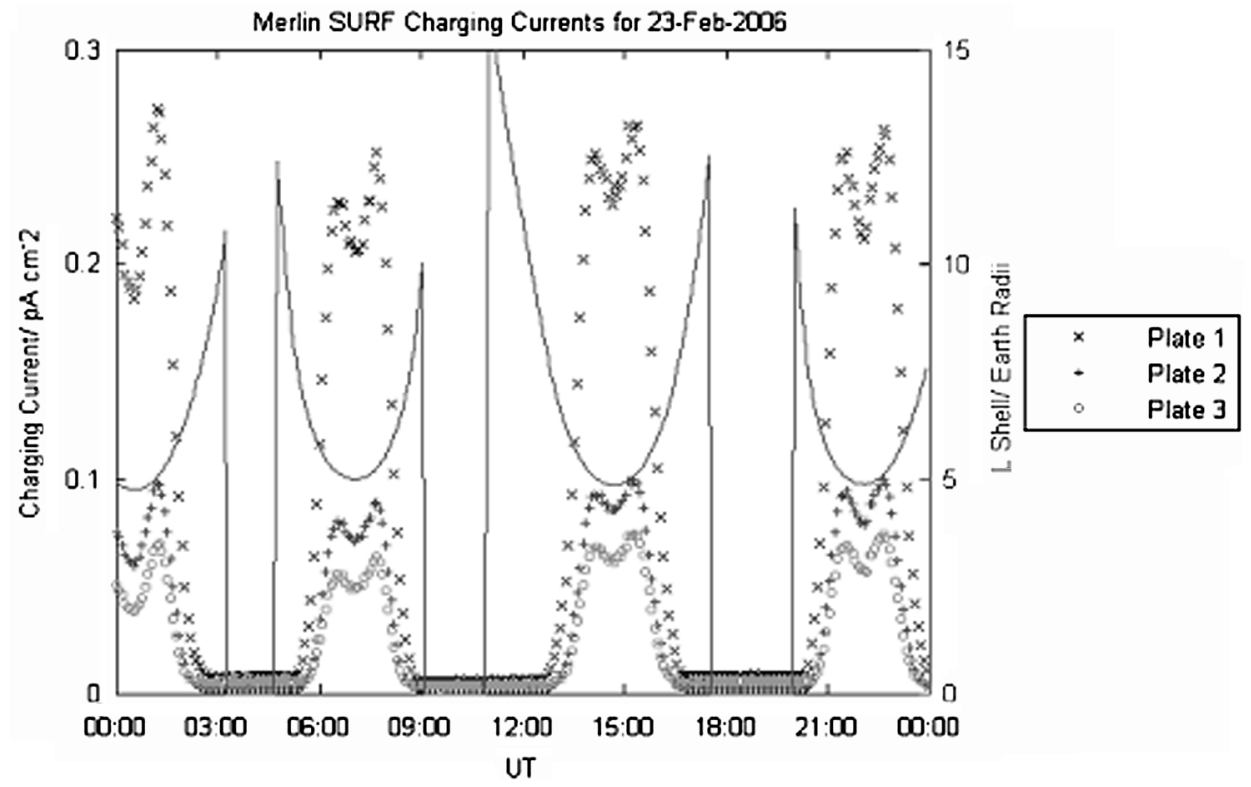

Fig. 5. The Merlin SURF Charging Currents plotted against Universal Time for 23 February 2006, overlaid with the McIlwain L shell Geomagnetic coordinate. All Currents have excellent signal to noise ratios and all register the crossings of the belts.

design has proven itself to be virtually immune to proton contamination, overcoming a drawback of many conventional electron detectors that can become contaminated during solar particle events.

\section{RADFETs}

Total dose is measured at two locations within the unit using calibrated RADFETs. These devices are left unbiased so that interruptions in power do not affect their readings. The shielding and sensitivity of the RADFETs is adjusted according to the mission. For Giove-A the shielding is $3 \mathrm{~mm}$ and $6 \mathrm{~mm}$ of equivalent Aluminium in the $+\mathrm{Z}$ (space facing) direction with greater shielding elsewhere-including the RADFET lids.

\section{PRELIMINARY DATA}

The Merlin and CEDEX monitors were both switched on soon after launch, and a near continuous dataset has been provided commencing at the beginning of January. A preliminary investigation of the data reveals that both instruments are working well and are detecting the magnitude and variability of the outer radiation belts.

The Heavy Ion telescope on CEDEX is monitoring the cosmic ray particle flux and data shows a good agreement with CREME86 predicted fluxes [5], as seen in Fig. 3. The particle flux is preliminarily assumed to be over $4 \pi$ with shielding of $10 \mathrm{~mm} \mathrm{Al}$. A $\pm 5 \mathrm{~mm}$ deviation produces a maximum error of $20 \%$ at high LET for the model predictions. 


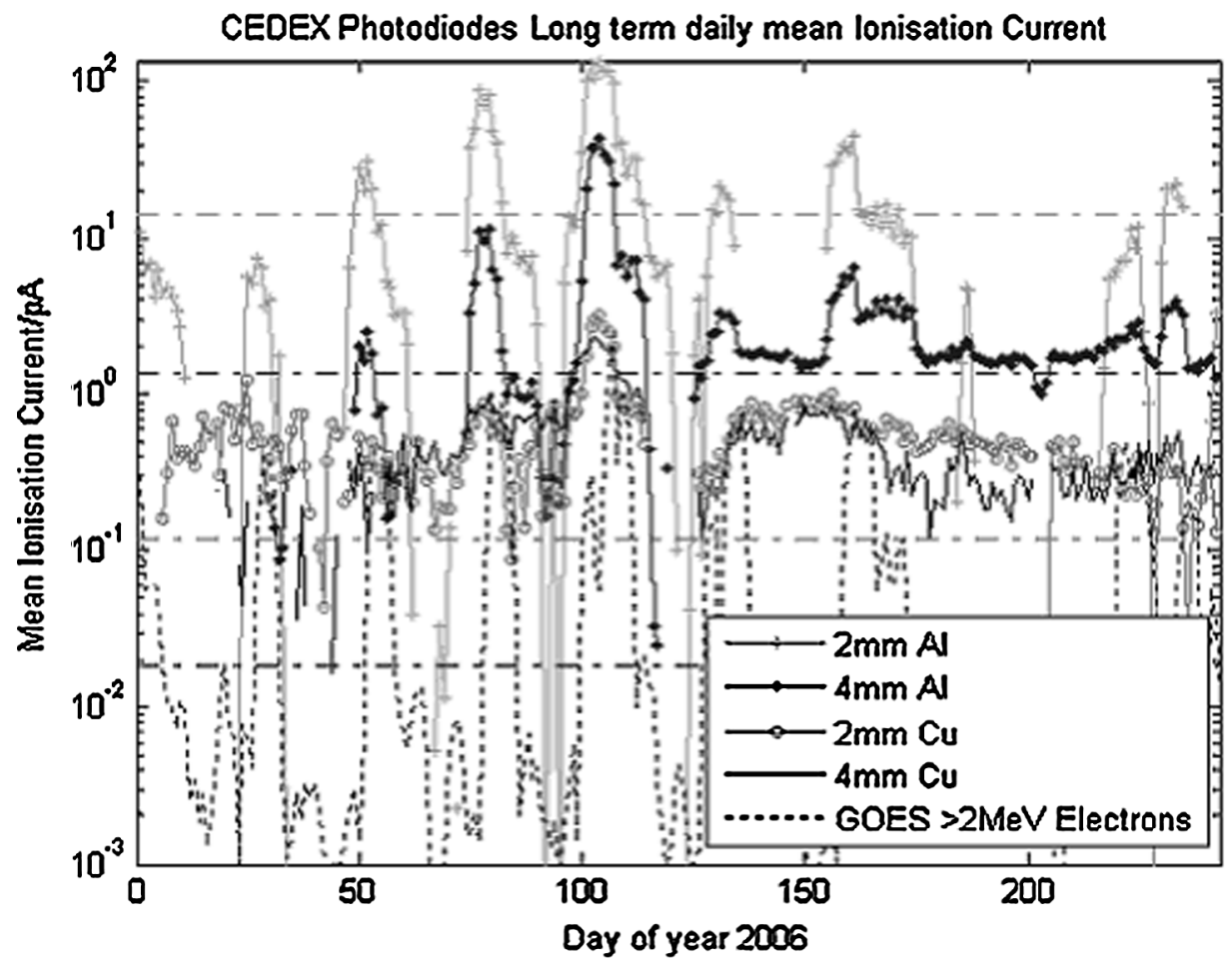

Fig. 6. The daily mean Ionisation current from the CEDEX experimental photo diodes from early January to late August 2006 with preliminary AE-8 model predictions for mean currents overlaid. The dynamics of the radiation belt are clearly shown in the $2 \mathrm{~mm}$ and $4 \mathrm{~mm}$ Al. channels, whereas the Cu. Channels are dominated by noise. Here, the $2 \mathrm{~mm}$ Al diode saturates at $410 \mathrm{pA}$. GOES $>2 \mathrm{MeV}$ Electron Flux is plotted in arbitrary units.

The experimental CEDEX photodiodes are registering the crossings of the belt. The lightest shielded diode $(2 \mathrm{~mm} \mathrm{Al})$ clearly shows the crossing of the radiation belts, as seen in Fig. 4. The passage of the radiation belt is not as clear in the $4 \mathrm{~mm} \mathrm{Al}$ shielded diode, but this is as expected as the radiation belt models indicate that the dose-rate from this photo-diode should be approximately a factor of 10 lower than the $2 \mathrm{~mm}$, or $\sim 10 \mathrm{pA}$. The belt crossings are clearly seen in the three Merlin SURF charging currents. All produce clear signals for belt crossings as seen in Fig. 5.

The time series data of the CEDEX photo diodes, seen in Fig. 6, show the strong variability of the outer radiation belt, registering the enhancement and subsequent decays of the belt over the period. A number of events are apparent, all of which coincide with enhancements detected in the GOES-12 $>2 \mathrm{MeV}$ daily fluence data at an L-shell of $6.6 \mathrm{R}_{\text {Earth }}$. The model predictions are based on basic 3D GRAS (GEANT-4 Radiation Analysis for Space) [6] Monte Carlo simulations using the AE-8 model spectrum for Solar Minimum, which is the standard model in engineering applications for trapped electrons [7].

The Merlin SURF plates are detecting significant rates of internal charging caused by the energetic electron environment at three different shielding depths. Large variations in charging rates are noted both around the orbit and also over longer timescales due to outer belt enhancements as seen in Fig. 7 . The model predictions are again based on basic 3D GRAS modelling of the energy response of the SURF plates using Monte Carlo simulations using the AE- 8 energy spectrum. The effect of the enhancement events is clearly seen in the Merlin

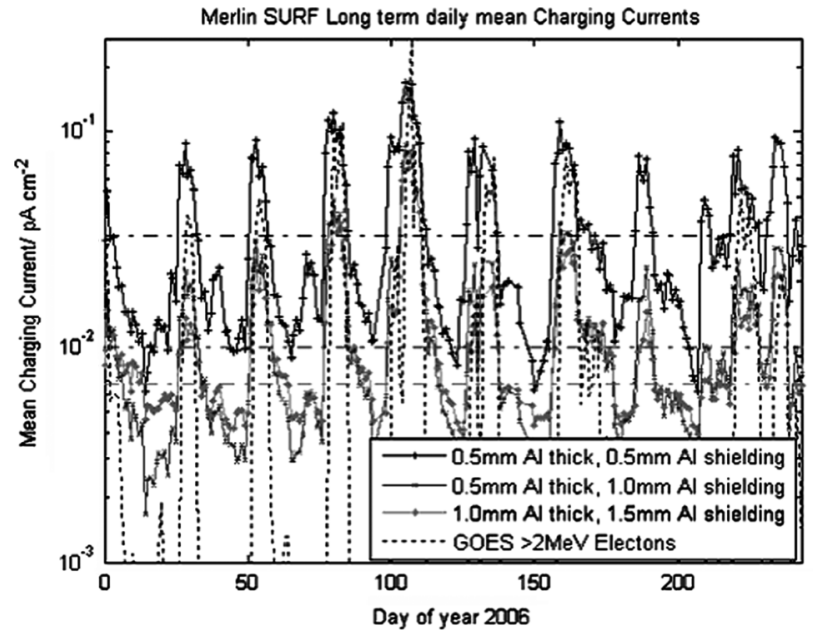

Fig. 7. The daily maximum charging current from the three SURF plates from early January to late August 2006 with preliminary AE-8 model predicted mean Charging Current for the SURF plates. GOES $>2 \mathrm{MeV}$ Electron Flux is plotted in arbitrary units.

RADFETs with dose-rate accumulation increasing during the events in Fig. 8.

\section{CONCLUSION}

Both Giove-A radiation monitors are functional and collecting data. A preliminary look at the data shows that the instruments are registering large variations in the state of the outer electron belt. This high variability is due to geomagnetic storm conditions triggered by interactions with solar wind 
Total Accumulated Dose for $3 \mathrm{~mm}$ Al shielded window RADFET
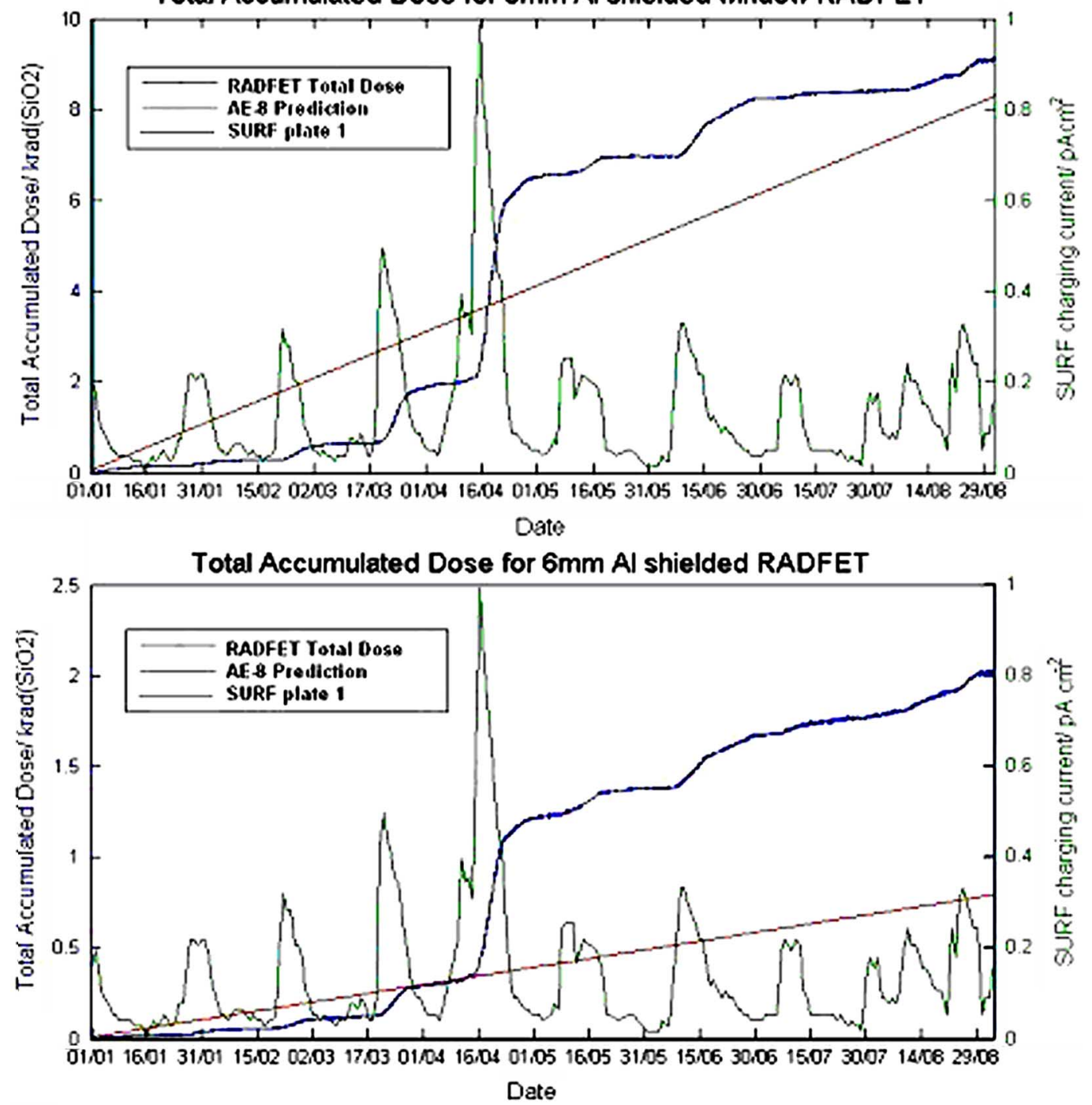

Fig. 8. The Accumulated Total Dose from the two Merlin RADFETs, clearly dominated by a reoccurring event indicated by SURF charging currents. The Total Dose is greater than predicted by AE-8/AP-8 due to periodic electron enhancements, primarily to a single event in April.

streams caused by a Coronal hole on the Sun. This causes changes in the intensity and spatial structure of the belt when the Coronal holes are magnetically directed at Earth. Coronal holes can be a persistent feature at this point in the solar cycle and indeed many of the events registered stem from a single coronal hole, rotating with the Sun giving a periodicity of $\sim 27$ days. These storm conditions and subsequent enhancements can also be triggered by Coronal Mass Ejections directed at the Earth, however to date no significant events of this nature have been observed.

The data thus far shows that ionising dose is delivered primarily in distinct surges during electron enhancement events triggered by interactions with Coronal holes on the Sun. Over the 8 months to date the dose measured in the RadFET at $3 \mathrm{~mm}$ $\mathrm{Al}$ eq. shielding ( $+\mathrm{z}$ direction) exceeds the prediction based on AE8 by only a small margin $(\sim 10 \%)$. The dose received by the more heavily shielded RADFET ( $6 \mathrm{~mm} \mathrm{Al} \mathrm{eq.} \mathrm{in}+\mathrm{z}$ direction) is higher than the AE8 prediction by a factor 2 . Since a large frac- tion of these doses have been received during a small number of electron enhancements, in particular the April 2006 event, the results are unlikely to be representative of the average conditions over a whole solar cycle. A longer period of observation will be needed to compare measured doses with those predicted by a long term average model such as AE-8.

The Cosmic ray spectrum measured by the CEDEX instrument telescope is in good agreement with CREME86 models with the interplanetary weather index set to take into account Galactic cosmic rays and the fully ionised component (i.e.,: CREME86 $\mathrm{M}=2$ ) and the $90 \%$ worst case cosmic ray level (i.e.,: CREME86 $\mathrm{M}=3$ ). The majority of the spectrum lies between the $\mathrm{M}=2$ and $\mathrm{M}=3$ CREME86 models.

Comparison of CEDEX Dose Rate Diodes to $>2 \mathrm{MeV}$ electron INTEGRAL/IREM count rate data from periods when the spacecraft was between L shells of 4.45 and 4.55 Earth radii produces a good empirical agreement with INTEGRAL/IREM data over time as seen in Fig. 9. 


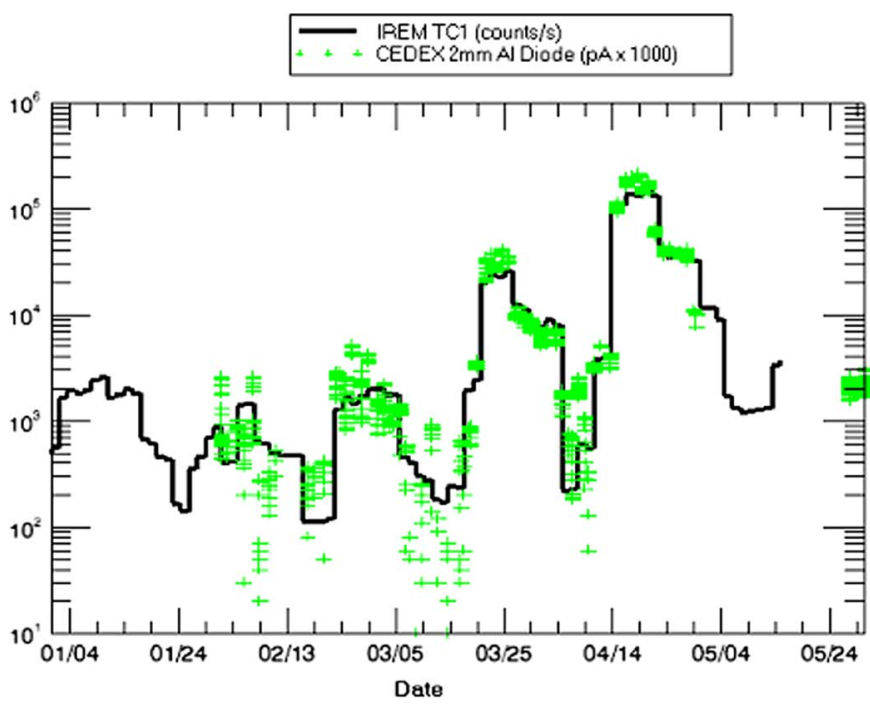

Fig. 9. INTEGRAL/IREM data for 1st January to 1st June 2006 for electrons $>2 \mathrm{MeV}$ and between 4.45 and 4.55 Earth Radii over-plotted with CEDEX 2 $\mathrm{mm} \mathrm{Al}$ Ionisation current $\times 1000$. Demonstrates good proportionality between the two datasets.

\section{REFERENCES}

[1] A. Mohammadzadeh, "The ESA standard radiation environment monitor program first results from PROBA-1 and INTEGRAL," IEEE Trans. Nucl. Sci., vol. 50, no. 6, pp. 2272-2277, Dec. 2003.

[2] C. I. Underwood, A. da Silva Curiel, and M. N. Sweeting, "In-orbit monitoring of 'space weather' and its effects on commercial-off-theshelf (COTS) electronics-A decade of research using micro-satellites," presented at the 53rd Int. Astronautical Congress, Houston, TX, Oct. 10-19, 2002, IAC-02-IAA.6.3.04.

[3] C. I. Underwood, A. da Silva Curiel, and M. Sweeting, "A doublepeaked profile of the inner radiation belt-First flight results from the AMSAT-OSCAR-40 cosmic-ray experiment," presented at the 53rd Int. Astronautical Congress, Houston, TX, Oct. 10-19, 2002, IAC-02IAA.11.1.02.

[4] K. Ryden, "The MERLIN space weather monitor and its planned flight on the Galileo system testbed satellite (GSTB-V2/A)," presented at the 55th Int. Astronautical Congress, Vancouver, BC, Canada, 2004, IAC-04-IAA.4.9.3/U.6.04.

[5] J. H. Adams, Jr., Cosmic Ray Effects on MicroElectronics NRL Memorandum Rep. 5901, 1986, Part IV.

[6] G. Santin, "GRAS: A general-purpose 3-D modular simulation tool for space environment effects analysis," IEEE Trans. Nucl. Sci., vol. 52, no. 6, pp. 2294-2299, Dec. 2005.

[7] J. I. Vette, The AE-8 Trapped Electron Model Environment, NSSDC/ WDC-A-R\&S 91-24, 1991a. 